\title{
THE
}

\section{User-Based Photometer Analysis of Effluent from Advanced Nitrogen-Removal Onsite Wastewater Treatment Systems}

\author{
Bianca N. Ross \\ University of Rhode Island \\ George W. Loomis \\ University of Rhode Island, gloomis@uri.edu \\ Kevin P. Hoyt \\ José A. Amador \\ University of Rhode Island, jamador@uri.edu
}

Follow this and additional works at: https://digitalcommons.uri.edu/nrs_facpubs

\section{The University of Rhode Island Faculty have made this article openly available.}

Please let us know how Open Access to this research benefits you.

This is a pre-publication author manuscript of the final, published article.

Terms of Use

This article is made available under the terms and conditions applicable towards Open Access Policy Articles, as set forth in our Terms of Use.

\section{Citation/Publisher Attribution}

Ross, B.N., Loomis, G.W., Hoyt, K.P. et al. User-Based Photometer Analysis of Effluent from Advanced Nitrogen-Removal Onsite Wastewater Treatment Systems. Water Air Soil Pollut 229, 389 (2018). https://doi.org/10.1007/s11270-018-4039-z Available at: https://doi.org/10.1007/s11270-018-4039-z

This Article is brought to you for free and open access by the Natural Resources Science at DigitalCommons@URI. It has been accepted for inclusion in Natural Resources Science Faculty Publications by an authorized administrator of DigitalCommons@URI. For more information, please contact digitalcommons-group@uri.edu. 


\title{
User-based Photometer Analysis of Effluent from Advanced Nitrogen-Removal Onsite Wastewater Treatment Systems
}

\author{
Bianca N. Ross*, George W. Loomis, Kevin P. Hoyt, and Jose A. Amador
}

*Corresponding author: Bianca N. Ross

E-mail: bpeixoto10@uri.edu

T: (401) 874-2902

Bianca N. Ross, Kevin P. Hoyt, Jose A. Amador

Affiliation/address: Laboratory of Soil Ecology and Microbiology, University of Rhode Island, 1 Greenhouse Rd., Kingston, RI 02881, USA

Bianca N. Ross:

E-mail: bpeixoto10@uri.edu

Kevin P. Hoyt:

E-mail: kevin_hoyt@uri.edu

Jose A. Amador

E-mail: jamador@uri.edu

George W. Loomis

Affiliation/address:

New England Onsite Wastewater Training Center, University of Rhode Island, 102 Coastal Institute, 1 Greenhouse Rd., Kingston, RI 02881, USA

E-mail: gloomis@uri.edu

Keywords: Onsite wastewater treatment system, wastewater, rapid test, photometer, nitrogen, regression analysis

\section{Acknowledgements}

This work was funded by the USDA National Institute of Food and Agriculture, Hatch Multi-State NE 1545 Project [accession number 1007770] and by a grant (00A000128-0) awarded by the U. S. Environmental Protection Agency to Jose Amador and George Loomis at the University of Rhode Island. We thank our regulatory partners at the Rhode Island Department of Environmental Management and the town of Charlestown, Rhode Island for assisting us with technical aspects of the project, as well as procuring study sites. We also thank the Charlestown homeowners who allowed us to study their advanced N-removal OWTS. Special thanks go to Matt Dowling, Alicia Boucher and Jonathan Ludovico for their technical 
and field support, and to Andrew Ross for assistance with the development of a cost-benefit analysis.

Although the information in this paper has been funded wholly or in part by the U. S. Environmental Protection Agency, it has not undergone the Agency's publications review process and therefore may not necessarily reflect the views of the Agency; no official endorsement should be inferred. The viewpoints expressed here do not necessarily represent those of the town of Charlestown, the Rhode Island Department of Environmental Management, or the U. S. Environmental Protection Agency, nor does mention of trade names, commercial products, or causes constitute endorsement or recommendation for use. 


\begin{abstract}
Advanced nitrogen-removal onsite wastewater treatment systems (OWTS) are used to reduce total nitrogen $(\mathrm{N})$ levels in domestic wastewater. Maintaining system performance requires regular monitoring and in situ rapid tests can provide an inexpensive option for assessing treatment performance. We used a portable photometer to measure ammonium and nitrate concentrations in final effluent from 46 advanced N-removal OWTS, sampling each site at least three times in 2017. To assess photometer accuracy, we compared measurements made using the photometer with those determined by standard laboratory methods using linear regression analysis and a two-tailed $t$-test to compare regression parameters to those for a perfect linear relationship $($ slope $=1$, intercept $=0$ ). Our results show that photometer-based analysis reliably estimates inorganic $\mathrm{N}$ (ammonium and nitrate) concentration in field and laboratory settings. Photometer-based analysis of the sum of inorganic N species also consistently approximated the total $\mathrm{N}$ concentration in the final effluent from the systems. A cost-benefit analysis indicated that the photometer is a more cost-effective option than having samples analyzed by commercial environmental testing laboratories after analysis of 8 to 33 samples. A portable photometer can be used to provide reliable, cost-effective measurements of ammonium and nitrate concentrations, and estimates of total $\mathrm{N}$ levels in advanced N-removal OWTS effluent. This method can be a viable tool for triaging system performance in the field, helping to identify systems that are not functioning properly and may need to be adjusted or repaired by an operation and maintenance service provider in order to meet treatment standards.
\end{abstract}




\section{Introduction}

Advanced nitrogen-removal onsite wastewater treatment systems (OWTS) are used to mitigate the impact of residential wastewater on ecosystems. Because nitrogen $(\mathrm{N})$ is a limiting nutrient in coastal watersheds, increased $\mathrm{N}$ inputs from wastewater promote eutrophication, which can result in fish and shellfish kills (Carpenter et al. 1998; Sohail and Adeloju 2016). Advanced N-removal OWTS aim to reduce N levels in wastewater by cycling it through an advanced treatment train that promotes successive nitrification (the conversion of ammonium to nitrate) and denitrification (the conversion of nitrate to nitrous oxide and/or dinitrogen gas), ultimately resulting in $\mathrm{N}$ being removed from the wastewater and emitted into the atmosphere (Fig. 1; Oakley et al. 2010).

Wastewater management and regulatory agencies rely on service providers - trained professionals responsible for carrying out operation and maintenance of OWTS, which can include quantifying tank substrate levels (sludge and scum), inspecting system electrical components, and evaluating system physical integrity (Bounds et al. 2004). Although measuring total $\mathrm{N}(\mathrm{TN})$ is not usually required during system maintenance, frequent monitoring of advanced OWTS TN concentrations in Cape Cod, MA has significantly improved system performance (BCDHE 2012). Previous efforts have assessed the efficacy of using rapid tests for field monitoring of advanced OWTS. Rapid tests allow for in situ analysis of system treatment performance, and can provide much of the same information as an external laboratory would, but faster and at less cost to the service provider (Bounds et al. 2004). Rapid tests for in situ measurement of $\mathrm{N}$ levels are particularly important for advanced OWTS because of the harmful threat that N-rich residential wastewater poses to coastal watersheds (Valiela et al. 1992). 
Service providers need efficient, inexpensive methods for evaluating N-removal performance of advanced OWTS.

$\mathrm{TN}$ is the sum of ammonium, nitrate, and organic $\mathrm{N}$. The few field tests capable of quantifying TN are time- and labor-intensive (Hach 2017; Hanna 2018), such that measuring ammonium and nitrate levels in the field may be a feasible alternative to quantifying TN. Several types of rapid field tests exist to measure the inorganic fraction of TN. Brannon et al. (2017) measured ammonium, nitrate, TN, and 5-day biochemical oxygen demand (BOD 5 ; a proxy for organic $\mathrm{N}$ ) in effluent from advanced OWTS. Based on these measurements, it appears that $\mathrm{BOD}_{5}$ does not significantly influence $\mathrm{TN}$ concentration in effluent with less than 19 $\mathrm{mg} / \mathrm{L} \mathrm{TN}$, while $\mathrm{BOD}_{5}$ does have a significant contributing influence on $\mathrm{TN}$ concentration in effluent with a TN concentration was higher than $19 \mathrm{mg} / \mathrm{L}$. Because organic $\mathrm{N}$ is unlikely to make up a significant fraction of the TN present in treated effluent of advanced systems, quantifying ammonium and nitrate levels could potentially be used as a proxy for TN levels in effluent. In addition, evaluating ammonium and nitrate levels may provide insight into how the OWTS is treating the wastewater. Extremely high or low concentrations of either inorganic $\mathrm{N}$ species may indicate the extent to which specific $\mathrm{N}$ transformations are occurring in the system. For example, a system producing effluent from the nitrification component of the treatment train that is high in ammonium and low in nitrate is likely not facilitating nitrification, which will ultimately impede system N-removal.

Test strips are commonly used to measure ammonium and nitrate concentrations in freshwater samples (Isbell et al. 2006; Murphy et al. 2014); however, few studies have investigated the efficacy of these tests with wastewater effluent. Lancellotti et al. (2016) measured ammonium and nitrate concentrations in effluent from advanced $\mathrm{N}$-removal systems in 
an outdoor (field) and indoor (laboratory) setting using both test strips and wet chemistry test kits and reported that ammonium and nitrate test strips only provide accurate results in the laboratory. A variety of factors - including contamination, temperature, precipitation, humidity, wind, and differences in ambient sunlight - can interfere with measurements that are not made in a controlled environment, one of the drawbacks of using field-based rapid tests (Taylor and Kerr 1941). Human bias may also lead to inaccurate rapid test results, especially for tests that require interpretation of color (Fellers et al. 2015).

One rapid field method for analysis of ammonium and nitrate in effluent that has not yet been evaluated is spectrophotometry. A portable photometer can be used to quantify ammonium and nitrate concentrations colorimetrically, based on the principle that light absorbance at a particular wavelength is directly proportional to the concentration of the compound analyzed (Harris 1991). Using a portable photometer to measure the concentration of $\mathrm{N}$ species removes the human bias associated with tests that require color interpretation and reduces interference of environmental factors. User manuals containing step-by-step instructions, as well as troubleshooting options and data management suggestions, help make photometer usage possible for OWTS operation and maintenance staff. Portable photometers and kits containing the reagents necessary for making these measurements are available commercially (Fig. 2).

To determine the feasibility of using a portable photometer to measure inorganic $\mathrm{N}$ levels in wastewater, we analyzed effluent from 46 advanced N-removal OWTS in Charlestown, Rhode Island. Twenty-four of the systems serve homes that are occupied year-round, while 22 systems serve seasonally-occupied homes. We analyzed effluent in field and laboratory settings using a portable photometer and analyzed the same samples using standard laboratory methods. We first compared measurements generated by standard methods with those obtained using the 
photometer in the field vs. in the laboratory setting to ascertain whether the test setting (outdoor vs. indoor) influences photometer results. To assess the accuracy of ammonium and nitrate measurements generated by the photometer, we then compared results obtained by the photometer to results obtained via standard methods. We also evaluated whether the sum of ammonium and nitrate measured with the photometer could predict TN measured using standard methods. Finally, we performed a cost-benefit analysis evaluating the cost-effectiveness of measuring ammonium and nitrate on the photometer vs. having samples analyzed for the same $\mathrm{N}$ species by commercial environmental testing laboratories.

\section{Methods}

\section{Study Systems}

We sampled final effluent from four different N-removal OWTS technologies: (i) Orenco Advantex® AX20, (ii) Orenco Advantex ${ }^{\circledR}$ RX30, (iii) BioMicrobics MicroFAST®, and Norweco Singulairß (models TNT, 960, and DN). We sampled from a total of 46 sites in Charlestown, Rhode Island. Twenty-four of the systems served homes occupied year-round, while 22 systems served seasonally-occupied homes. Detailed descriptions of the systems can be found in the Supplementary Materials.

\section{Sample Collection}

Final effluent samples (effluent to be dispersed to the drainfield) were collected in June, September, and December 2017 for systems serving homes occupied year-round, and in June, July, August, and September for systems serving seasonally-occupied homes. The Advantex systems were sampled at the recirculating splitter valve assembly, while the FAST and Singulair technologies were sampled from the drainfield pump basin. A grab sample was collected into a 
clean 1-L plastic bottle. Part of the sample was used for field analysis, and the remainder of the sample was stored at $4^{\circ} \mathrm{C}$ until transported to the laboratory (within 8 hours of sampling). Upon arrival at the laboratory, $25 \mathrm{~mL}$ of sample was passed through a $0.45-\mu \mathrm{m}$-pore-size membrane filter and the filtrate was frozen until analyzed for ammonium and nitrate using standard methods (described below).

\section{Analyses}

\section{$\underline{\text { Standard Methods }}$}

The concentration of ammonium (range of 0-5 $\mathrm{mg} \mathrm{NH}_{4}{ }^{+}-\mathrm{N} / \mathrm{L}$; Weatherbern 1967) and nitrate (range of 0-2 mg $\mathrm{NO}_{3}{ }^{-}-\mathrm{N} / \mathrm{L}$; Doane and Horwath 2003) was determined colorimetrically using a BioTek Synergy HTX multi-mode microplate reader (Winooski, Vermont). Total N concentration was determined using the persulfate oxidation method (APHA 1998), and the resulting nitrate measured colorimetrically using the microplate reader. Effluent samples were diluted with deionized distilled water as necessary so that they would fall within the detection range.

Data collected using standard methods were required to meet certain performance criteria in order to ensure that results complied with established standards of accuracy and precision. The calibration curve for each plate was required to have an $R^{2}$ of at least 0.99 . All samples were analyzed in triplicate and were reanalyzed if the coefficient of variation among the triplicates was greater than $20 \%$. The dilution factor of samples that measured out of range of the calibration curve was adjusted and samples were reanalyzed. Method blanks analyzed for ammonium could not exceed $200 \mu \mathrm{g} \mathrm{NH}{ }_{4}{ }^{+}-\mathrm{N} / \mathrm{L}$, blanks analyzed for nitrate could not exceed 100 $\mu \mathrm{g} \mathrm{NO}{ }^{-}-\mathrm{N} / \mathrm{L}$, and blanks analyzed for $\mathrm{TN}$ could not exceed $50 \mu \mathrm{g} \mathrm{N} / \mathrm{L}$. A laboratory control 
standard was also analyzed with the effluent samples and its ammonium, nitrate, and TN concentrations could not deviate more than $20 \%$ from the established values. One field duplicate per 10 sites was analyzed for ammonium, nitrate, and $\mathrm{TN}$, and the relative percent difference between the field duplicate and original sample could not exceed $100 \%$.

\section{$\underline{\text { Photometer Method }}$}

Ammonium and nitrate concentrations were measured with a photometer in field and laboratory settings using a model 83214 Hanna Instruments Multiparameter Bench Photometer (Woonsocket, Rhode Island). For both the ammonium and nitrate analyses, a $5 \mathrm{~mL}$ syringe was used to add $1 \mathrm{~mL}$ of unfiltered effluent to the test vials. Once effluent had been added to a vial, it was inverted several times, inserted into the photometer, and read as a blank. The test-specific reagent was then added to the vial and the vial was inverted several times and inserted into the photometer. The photometer has a built-in timer programmed for each test, and determined the concentration after the required amount of time had passed. Ammonium was determined using the Nessler method, in which 4 drops of Nessler's reagent (dipotassium tetraiodomercurate (II) in dilute $\mathrm{NaOH}$ ) were added to each test vial, which reacts with ammonium to produce a yellowbrownish color (Jeong et al. 2013; Hanna Instruments 2016). Concentrations of up to $100 \mathrm{mg}$ $\mathrm{NH}_{4}{ }^{+}-\mathrm{N} / \mathrm{L}$ can be detected using this method. Nitrate concentration was measured using the chromotropic acid method, in which a powdered reagent (chromotropic acid disodium salt and sodium metabisulfite) is added to a test vial and reacts with sulfuric acid and nitrate in the effluent to produce a yellowish color (Sims and Jackson 1971; Hanna Instruments 2016). A nitrate concentration of up to $30 \mathrm{mg} \mathrm{NO}_{3}{ }^{-} \mathrm{N} / \mathrm{L}$ can be measured using this method. Photometer measurements were made in the field during the months of June, July, and August 2017. Samples obtained in September and December were kept in the dark at $4^{\circ} \mathrm{C}$ and analyzed with 
the photometer in the laboratory within 24 hours of collection. One field duplicate per 10 sites was analyzed for ammonium and nitrate, and the relative percent difference between the field duplicate and original sample could not exceed $100 \%$.

\section{$\underline{\text { Statistical Analyses }}$}

Linear regression analysis was used to evaluate the accuracy of measurements made with the photometer. Because the upper limits for detection using the photometer were $100 \mathrm{mg} \mathrm{NH}_{4}^{+}-$ $\mathrm{N} / \mathrm{L}$ and $30 \mathrm{mg} \mathrm{NO}{ }^{-}-\mathrm{N} / \mathrm{L}$, samples with values above this threshold (as determined using standard methods) were excluded from the data set. We used the values obtained using the photometer method as the dependent variable and values generated by standard laboratory methods as the independent variable. First, we assessed the influence of test setting (field vs. laboratory) on photometer-based measurements by comparing regression lines for each setting. A two-tailed $t$ test $(\alpha=0.01)$ was then used to identify which regression parameters (slope and intercept) differed between settings. To assess accuracy of measurements using the photometer, we compared ammonium and nitrate regression lines to a line representing the ideal relationship between concentrations measured with the photometer and with standard methods, with a yintercept of 0 , a slope of 1 , and an $R^{2}$ value of 1 . A two-tailed $t$ test $(\alpha=0.05)$ was used to identify regression parameters that deviated significantly from the ideal values for slope and intercept. For our analysis, a two-tailed $t$ test with $\alpha=0.05$ is a more stringent test than one with $\alpha=0.01$, because utilizing an $\alpha$ of 0.05 generates a smaller confidence interval for each regression parameter, narrowing the window of comparison to the ideal regression parameters. We also investigated the validity of estimating $\mathrm{TN}$ concentrations from the sum of ammonium and nitrate concentrations measured by the photometer compared to the same sum generated by standard methods by performing a linear regression using the sum of ammonium and nitrate 
values as the independent variable, and the corresponding $\mathrm{TN}$ value obtained via standard methods as the dependent variable.

\section{$\underline{\text { Cost-benefit Analysis }}$}

A cost-benefit analysis was performed to compare the cost of analyzing effluent samples for ammonium and nitrate using a photometer with the cost for the same analyses performed by two commercial environmental testing laboratories in Rhode Island (Lab A and Lab B). We considered the fixed and variable costs associated with both methods of analysis. For the photometer analysis, the fixed costs include the photometer and miscellaneous operational supplies (scissors and syringes). Because sending samples to a commercial laboratory does not require purchasing any instruments or supplies, this method has no fixed costs associated with it. Variable photometer costs consist of the cost of the reagents required to run ammonium and nitrate analyses, as well as the cost of reagent disposal, since hazardous waste disposal is required for the ammonium tests due to the use of Nessler's reagent, which contains mercury (N. Paterson, personal communication, April 24, 2018). These costs are variable because they depend on how many samples require analysis. The variable costs for commercial analysis are determined by each laboratory, and are incorporated in the rate charged per sample analyzed for a particular analyte.

\section{Results and Discussion}

Analysis of accuracy in a field vs. laboratory setting

To assess the photometer's ability to accurately measure effluent ammonium and nitrate concentrations, we performed a linear regression that compared the concentrations obtained via standard methods (independent variable) to those obtained by the photometer method (dependent 
variable) and compared the regression to that of an ideal 1:1 relationship between the two variables. Our first consideration was whether the external environment influenced photometer accuracy. To assess this, we performed separate regressions for ammonium and nitrate based on photometer measurements determined in the field, and then performed the same regressions for ammonium and nitrate values generated by the photometer in the laboratory in order to investigate the potential benefits of performing the analysis in a climate-controlled environment. We then conducted a two-tailed $t$ test $(\alpha=0.01)$ to determine whether the regression parameters differed between values obtained in field and laboratory settings for ammonium and nitrate.

When plotted against each other, the regression lines were very similar for ammonium concentrations measured using the photometer in field and in laboratory settings (Fig. 3). The nearly identical regression parameters observed suggest that the setting of this test (indoor vs. outdoor environments) does not significantly impact the photometer measurements (Table 1). The slope of the regression of the values determined in the laboratory did not differ significantly from that of the values determined in the field. The regression intercept for the field-based photometer measurements $\left(1.51 \mathrm{mg} \mathrm{NH}_{4}{ }^{+}-\mathrm{N} / \mathrm{L}\right)$ was slightly higher than that generated by labbased measurements $\left(0.67 \mathrm{mg} \mathrm{NH}_{4}{ }^{+}-\mathrm{N} / \mathrm{L}\right)$. The $99 \%$ confidence interval for field intercept $(0.57$ $-2.46)$ and laboratory intercept $(0.02-1.31)$ do overlap, suggesting minimal differences in the data.

The regression parameters for our comparison of nitrate concentrations determined using the photometer in the field and laboratory were nearly identical (Fig. 3; Table 1). Neither the slope nor the intercept of the laboratory-obtained values differed significantly from that of the field-obtained values, indicating that the photometer can be used to measure nitrate in both the field and laboratory settings. Based on the minimal effects of environment setting on ammonium 
measurements and the lack of effects of setting on nitrate measurements, we grouped photometer measurements made in the field and laboratory settings for subsequent analyses.

\section{Comparison of photometer method accuracy for ammonium vs. nitrate}

When ammonium values obtained using the standard method were plotted against those obtained using the photometer method, the resulting regression line was nearly identical to the 1:1 line representing the ideal relationship between the two methods (Fig. 4). This highlights the photometer's capability for accurately measuring ammonium. The regression line resulting from a comparison of nitrate values obtained using standard methods to those obtained using the photometer method deviated slightly from the 1:1 line (Fig. 4).

Analysis of the nitrate model's regression residuals showed that the values are not normally distributed; rather, it skews positively. This suggests that the photometer tends to overestimate effluent nitrate concentration. The difference may be due to differences in how effluent samples are processed prior to analysis. Analysis by the standard method requires that samples be filtered prior to analysis, whereas samples are not filtered prior to analysis by the photometer method. The measurement of ammonium and nitrate concentrations using the photometer is based on Beer's Law $(A=\varepsilon b c)$, which states that absorbance is directly proportional to the concentration of the compound for which the sample is being analyzed (where $\mathrm{A}=$ absorbance, $\varepsilon=$ molar absorptivity, $\mathrm{b}=$ path length, and $\mathrm{c}=$ concentration). Effluent contains organic and inorganic particles which block the light's path through the effluent, and is measured as the light being absorbed. This reduced transmittance can result in the photometer reporting a higher concentration than the sample actually has (Harris 1991). 
We conducted a two-tailed $t$ test $(\alpha=0.05)$ to determine which of the regression parameters generated by our comparison of photometer method and standard method measurements could be corresponded with ideal regression parameters (Table 2). Although the slope of the ammonium test's regression line was the only value deemed statistically similar to the regression parameters of a 1:1 line, the slope generated by the nitrate test regression was also close to the acceptable range of values. Intercepts for both the ammonium and nitrate regressions were greater than 0 , indicating that the photometer test may overestimate effluent ammonium and nitrate concentration of the analytes, likely due to the presence of particles and their effect on photometer absorbance calculations. Mathematically calculating the offset between photometer and standard method measurements and incorporating this calculated offset value into the photometer measurements may improve their accuracy.

\section{$\underline{\text { Sum of ammonium and nitrate as a predictor of total } \mathrm{N}}$}

We also examined whether the sum of ammonium and nitrate could predict effluent TN levels accurately. We compared the ability of the photometer and standard methods to predict effluent TN by summing the ammonium and nitrate values obtained by each method, plotting the sum against the TN content, and performing a regression analysis for each dataset (Fig. 5).

The sum of ammonium and nitrate generated by both the photometer and standard methods was strongly correlated with TN concentration (Table 3). Nevertheless, because organic $\mathrm{N}$ is also a component of the total amount of $\mathrm{N}$ in wastewater, quantifying only the inorganic forms of $\mathrm{N}$ cannot, in theory, predict TN levels accurately. Approximately $58 \%$ of sampling sites reported an average $\mathrm{BOD}_{5}$ value greater than $0 \mathrm{mg} / \mathrm{L}$, indicating that organic $\mathrm{N}$ is at least a minor contributor to $\mathrm{TN}$ levels for some sites. The regression line generated by the photometer analysis has a steeper slope than that of the standard methods regression line (Table 
3). The difference may be due to the fact that sample filtration is required for standard method analysis of ammonium and nitrate, while filtration is not required prior to analyzing samples with the photometer. As stated earlier, both organic and inorganic $\mathrm{N}$ species are present in wastewater in particulate form. Organic $\mathrm{N}$ can take the form of both macroscopic and microscopic particles. Because the photometer measures light absorbance, particles blocking the light's path through the sample could be misconstrued as sample absorbance. Thus, the presence of organic $\mathrm{N}$ could cause the photometer to slightly overestimate TN concentrations for effluent containing an appreciable amount of organic $\mathrm{N}$ in particulate form. The photometer method's tendency to overestimate effluent nitrate concentrations could also contribute to an overestimating TN measurements. The photometer method's overestimation of TN contrasts with our expectation that the method would underestimate $\mathrm{TN}$, since it only quantifies inorganic $\mathrm{N}$, effectively excluding the organic component of TN. These opposing factors, combined with the overall strength of the model ( $\mathrm{p}<0.001$ and $\left.R^{2}=0.68\right)$, suggest that the photometer is capable of reliably approximating TN concentrations. In a practical application, the sum of inorganic $\mathrm{N}$ measured with the photometer can be used to identify systems that are clearly not meeting performance standards, as well as those that should be targeted for more detailed analysis - those with an inorganic $\mathrm{N}$ concentration that is approaching or exceeding regulatory threshold values.

\section{Cost-benefit Analysis}

After considering the fixed and variable costs associated with analyzing samples on the photometer and sending samples to be analyzed by commercial environmental testing laboratories, we set up a linear function for each method and calculated the break-even point: the number of samples for which using the photometer would begin saving money (Table 4). The 
break-even point for ammonium analysis ranged from 27 to 33 samples. Because costs for nitrate analysis in a commercial laboratory are even higher, the photometer proved to be the more cost-effective option after having 8 samples analyzed by Lab A, and 20 samples analyzed by Lab B. Because Lab A does not actually offer individual nitrate testing (rather, nitrate is measured as a component of their TN analysis), the cost per sample is significantly higher than that offered by Lab B. Because we found that TN levels can be estimated from the sum of ammonium and nitrate determined using the photometer, we also calculated the break-even point for TN, which is 8 samples for Lab A and 11 samples for Lab B.

\section{Conclusions}

Our results show that that analysis using a portable photometer can be a viable method for measuring inorganic $\mathrm{N}$ levels in effluent, and users can operate the photometer in both indoor and outdoor settings without compromising accuracy. The photometer method can predict ammonium concentrations with great accuracy, and although it sometimes overestimates nitrate concentrations, it can also approximate nitrate concentrations. Reliable photometer ammonium and nitrate measurements can also provide valuable information about the systems' performance and capacity for facilitating $\mathrm{N}$ cycle processes. We also found that the sum of ammonium and nitrate measurements made using the photometer can be used as a proxy for $\mathrm{TN}$ concentration in effluent. This value can be used to help identify systems that are not performing optimally.

The photometer-based analysis of ammonium, nitrate, and TN is also a more financiallyviable option than sending samples to be analyzed at a commercial laboratory. Not only does it become the most cost-effective option after a small number of samples, the photometer method provides users, service providers, and regulatory decision makers with immediate information 
about system performance, vs. having to wait for a commercial laboratory to return analysis results (typically a turnaround time of 2-3 weeks). Our results have the potential to benefit researchers, service providers, and operation and maintenance staff in the advanced OWTS community. Utilizing the photometer will not only save them money, but it will also allow service providers to quickly monitor system performance, diagnose system problems, and facilitate real time adjustments to the system to help it meet treatment standard goals. This would provide regulatory agencies with added assurances that compliance is being met to help meet public and environmental health objectives. 
Table 1. Parameters of regressions comparing photometer accuracy in measuring ammonium and nitrate in field and laboratory settings $(n=54-64)$. A two-tailed $t$ test $(\alpha=0.01)$ was used to determine if test setting significantly influenced measurements reported. Values in bold differ significantly between settings. $R^{2}$ values were not included in this deviation analysis.

\begin{tabular}{lllll}
\hline Property & Test setting & \multicolumn{3}{c}{ Regression parameters } \\
\cline { 3 - 5 } & & Intercept & Slope & $R^{2}$ \\
\hline Ammonium & Field & $\mathbf{1 . 5 1}$ & 0.99 & 0.93 \\
& Laboratory & 0.67 & 1.01 & 0.98 \\
Nitrate & Field & 3.49 & 0.83 & 0.55 \\
& Laboratory & 5.13 & 0.79 & 0.51 \\
& & & & \\
\hline
\end{tabular}


Table 2. Parameters of regressions comparing photometer accuracy in measuring ammonium ( $n$ $=118)$ and nitrate $(n=115)$ concentrations to standard methods. Samples measured by the photometer both in the field and laboratory settings were considered in this analysis. A twotailed $t$ test ( $\alpha=0.05$ ) was used to determine which values differed significantly from ideal regression parameters ( 0 for intercept, 1 for slope). Values in bold are significantly different from ideal parameters.

\begin{tabular}{llcc}
\hline \multirow{1}{*}{ Property } & \multicolumn{3}{c}{ Regression parameters } \\
\cline { 2 - 4 } & Intercept & Slope & $R^{2}$ \\
\hline Ammonium & $\mathbf{1 . 1 2}$ & 1.00 & 0.95 \\
Nitrate & $\mathbf{4 . 3 2}$ & $\mathbf{0 . 8 1}$ & 0.52 \\
\hline
\end{tabular}


Table 3. Parameters of regressions investigating the ability to predict TN content based on the sum of ammonium and nitrate levels obtained via the photometer $(n=126)$ vs. standard methods $(n=122)$.

\begin{tabular}{|c|c|c|c|c|}
\hline \multirow[t]{2}{*}{ Method of analysis } & \multicolumn{4}{|c|}{ Regression parameter } \\
\hline & Intercept & Slope & $R^{2}$ & $\mathrm{p}$-value \\
\hline Photometer & 0.62 & 1.01 & 0.68 & $<0.001$ \\
\hline Standard & 5.86 & 0.86 & 0.64 & $<0.001$ \\
\hline
\end{tabular}


Table 4. Comparison of the cost-effectiveness of analyzing effluent samples with a portable photometer vs. sending samples to commercial environmental testing labs to be analyzed.

\begin{tabular}{|c|c|c|c|}
\hline $\begin{array}{l}\text { Method of } \\
\text { analysis } \\
\end{array}$ & Parameter & Fixed cost & $\begin{array}{l}\text { Variable cost } \\
\text { per sample }\end{array}$ \\
\hline \multirow[t]{3}{*}{ Photometer } & Ammonium & $\$ 560$ & $\$ 2.87$ \\
\hline & Nitrate & $\$ 560$ & $\$ 1.14$ \\
\hline & Total nitrogen & $\$ 560$ & $\$ 4.01$ \\
\hline \multirow[t]{3}{*}{ Lab A } & Ammonium & - & $\$ 24.00$ \\
\hline & Nitrate & - & $\$ 76.00$ \\
\hline & Total nitrogen & - & $\$ 76.00$ \\
\hline \multirow[t]{3}{*}{ Lab B } & Ammonium & - & $\$ 20.00$ \\
\hline & Nitrate & - & $\$ 30.00$ \\
\hline & Total nitrogen & - & $\$ 60.00$ \\
\hline
\end{tabular}




\section{Figures}

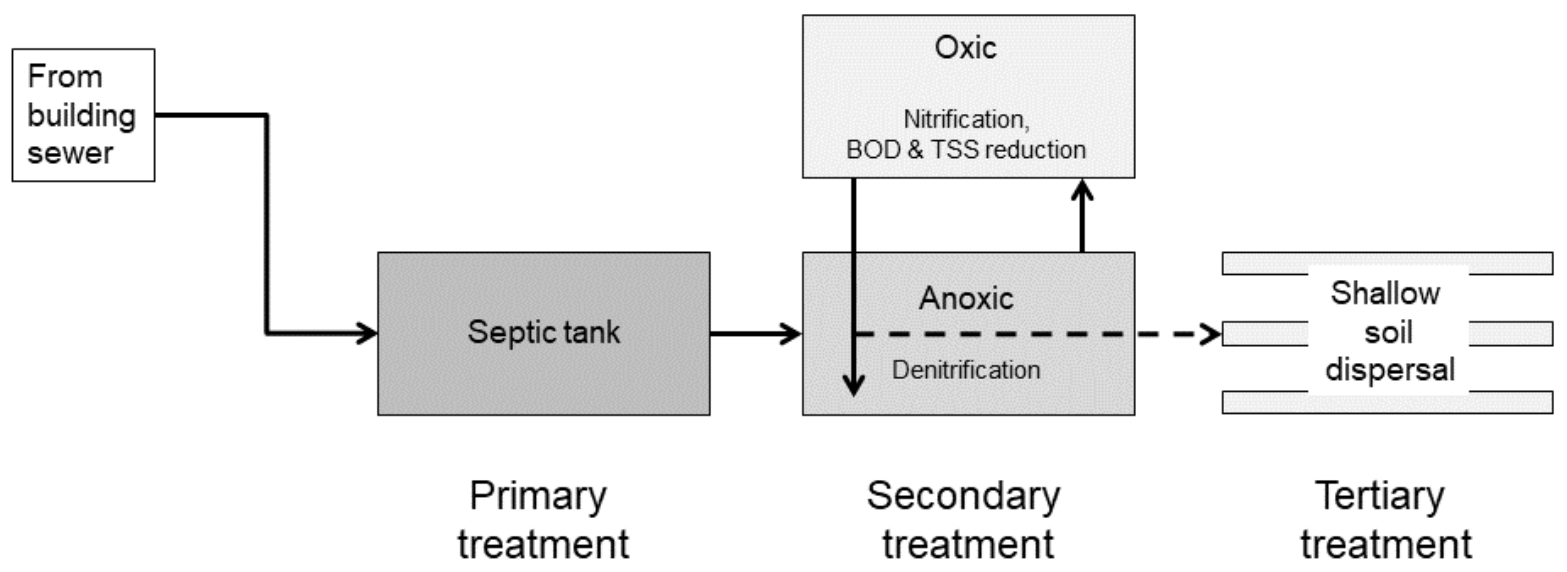

Fig. 1 Schematic diagram of advanced N-removal onsite wastewater treatment systems, illustrating components, treatment type, and treatment processes. Components are shown separately for clarity, but can also exist within one multi-compartment tank (BOD refers to biochemical oxygen demand; TSS refers to total suspended solids) 


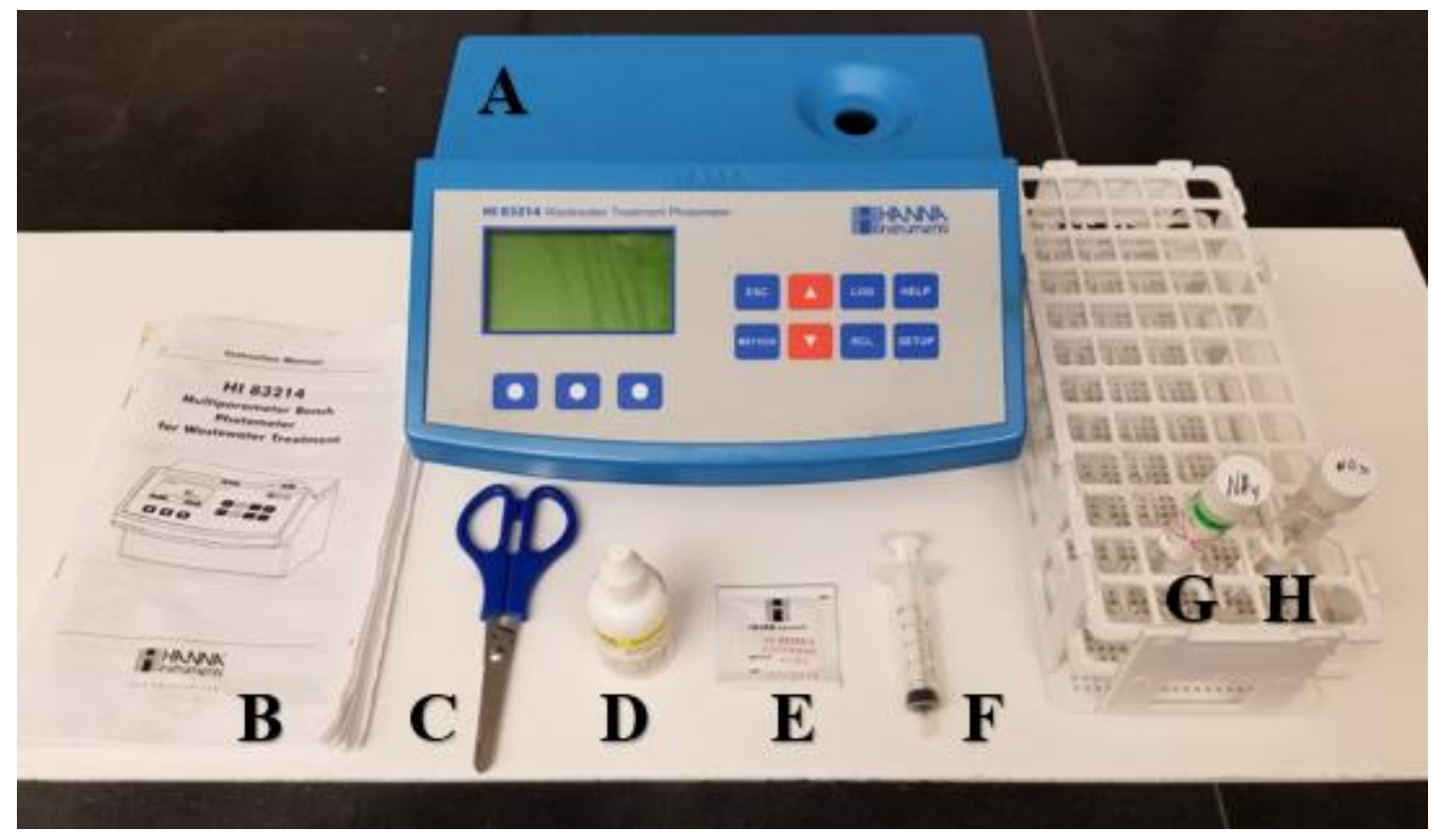

Fig. 2 Multiparameter bench photometer and supplies: photometer (A), instruction manual (B), scissors $(C)$, Nessler's reagent $(D)$, powdered reagent $(E)$, syringe $(F)$, ammonium test vial $(G)$, and nitrate test vial $(\mathrm{H})$ 

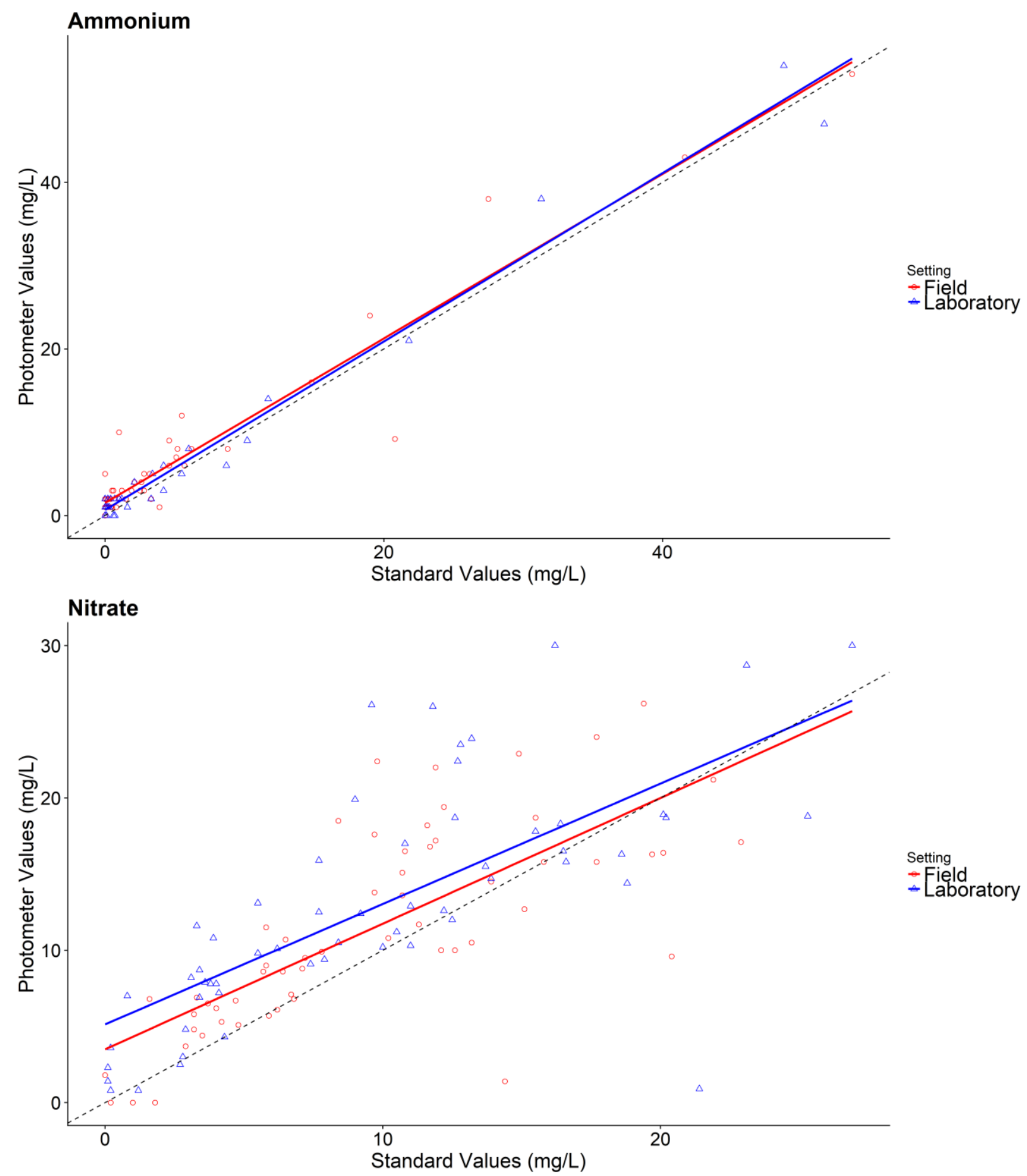

Fig. 3 Regression analyses of the accuracy of photometer ammonium and nitrate measurements made in the field vs. in the laboratory setting (illustrated by the solid lines). The dashed line represents the ideal 1:1 relationship between measurements obtained via the photometer and standard methods (with a slope of 1 and an intercept of 0 ) 

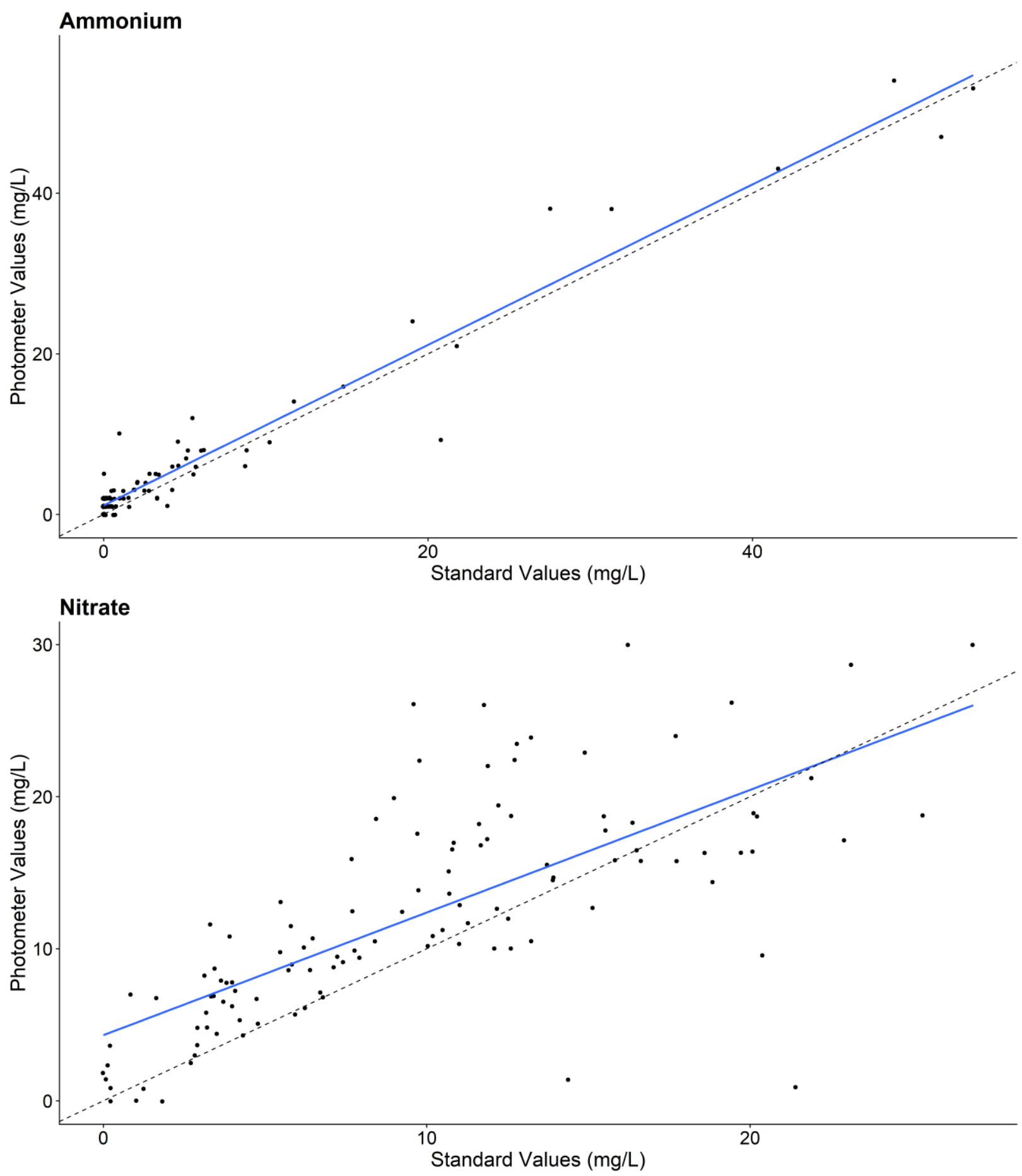

Fig. 4 Regression analyses of ammonium and nitrate concentration measurements made using photometer and standard methods (illustrated by the solid lines). The dashed line represents the ideal 1:1 relationship between measurements obtained via the photometer and standard methods (with a slope of 1 and an intercept of 0 ) 


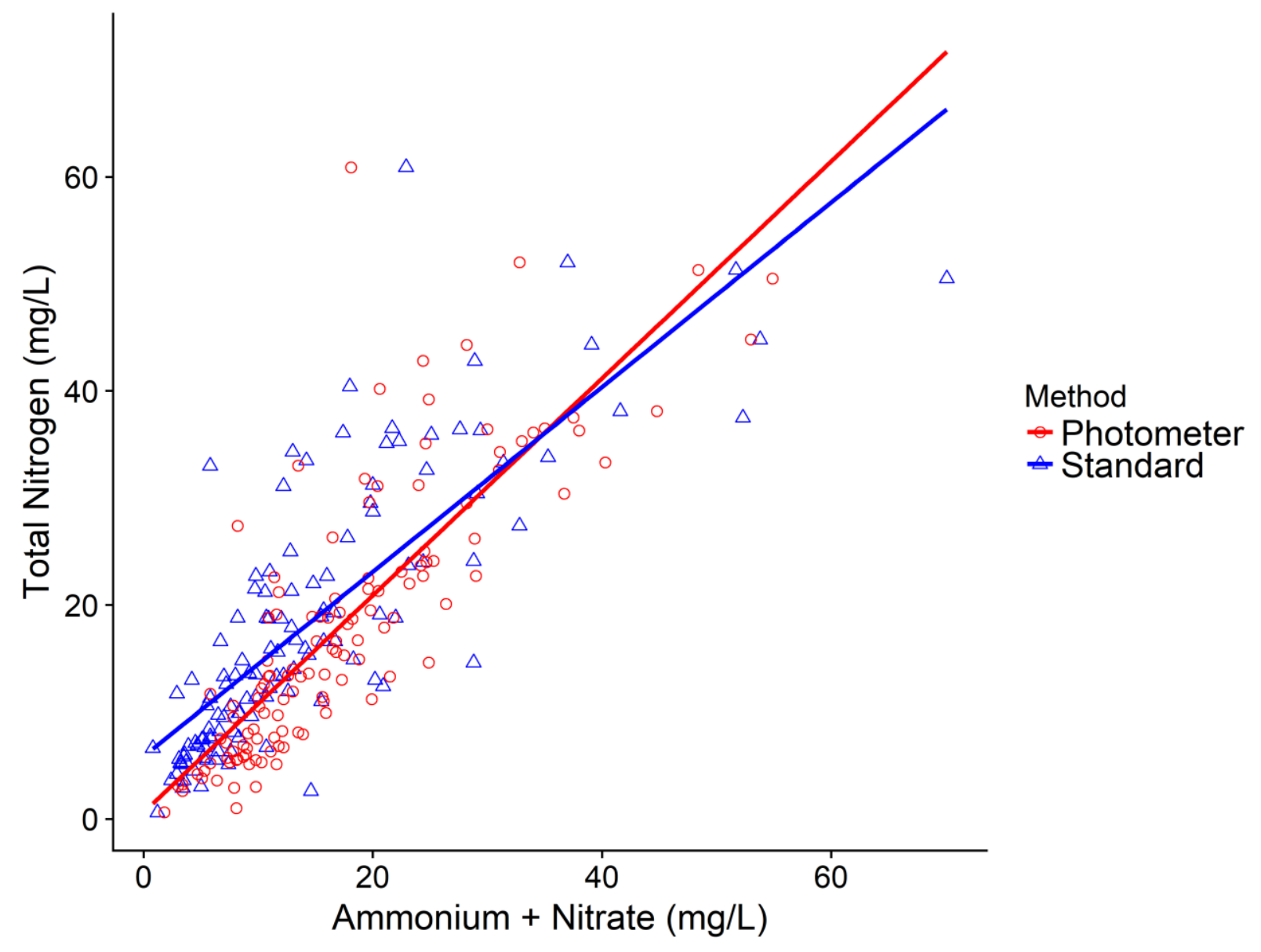

Fig. 5 Regression analysis of the relationship between TN and the sum of ammonium and nitrate values generated by either the photometer or standard methods 


\section{References}

American Public Health Association, \& American Water Works Association (APHA). (1998). Standard methods for the examination of water and wastewater, 20, 457-459.

BCDHE. Barnstable County Department of Health and Environment. (2012). Innovative/alternative septic system tracking. Available at: http://www.barnstablecountyhealth. org/programs-and-services/ia-septic-system-tracking.

Bounds, T., Denn, G., \& Bounds, N. T. (2004). Maintaining and troubleshooting advanced onsite systems: git'er done! 13th Technical Education Conference and Exposition, National Onsite Wastewater Recycling Association. Albuquerque, NM.

Brannon, E. Q., Moseman-Valtierra, S. M., Lancellotti, B. V., Wigginton, S. K., Amador, J. A., McCaughey, J. C., Loomis, G. W. (2017). Comparison of $\mathrm{N}_{2} \mathrm{O}$ emissions and gene abundances between wastewater nitrogen removal systems. Journal of Environmental Quality, 46, 931-938.

Carpenter, S. R., Caraco, N. F., Correll, D. L., Howarth, R. W., Sharpley, A. N., \& Smith, V. H. (1998). Nonpoint pollution of surface waters with phosphorus and nitrogen. Ecological Applications, 8(3), 559-568.

Doane, A., \& Horwath, W. (2003). Spectrophotometric determination of nitrate with a single reagent. Analytical Letters, 36(12), 2713-2722.

Fellers, T., Davidson, M., \& Spring, K. (2015). Human vision and color perception. Molecular Expressions. Available at: http://micro.magnet.fsu.edu/primer/lightandcolor/humanvisionintro.html

Jeong, H., Park, J., Kim, H. (2013). Determination of $\mathrm{NH}_{4}{ }^{+}$in environmental water with interfering substances using the modified Nessler method. Journal of Chemistry, 2013, 9 pages.

Hach. (2017). Total Nitrogen Reagent Set, HR, TNT. Available at: https://www.hach.com/totalnitrogen-reagent-set-hr-tnt/product?id=7640209860.

Hanna Instruments. (2016). Instruction Manual: Multiparameter Bench Photometer for Wastewater Treatment. Available at: https://hannainst.com/downloads/dl/file/id/2161/man83214_04_16.pdf.

Hanna Instruments. (2018). Total Nitrogen High Range Reagents with Barcode Recognition. Available at: https://hannainst.com/total-nitrogen-high-range-reagents-with-barcoderecognition-hi94767b-50.html.

Harris, D. C. (1991). Quantitative Chemical Analysis ( $3^{\text {rd }}$ ed.). New York, United States of America: W. H. Freeman and Company. 
Isbell, F., Mercer, D., Iqbal, M. (2006). Reliability of water quality test strips for field measurement of nutrients essential for developing mosquito larvae. American Journal of Undergraduate Research, 5(2), 1-8.

Lancellotti, B. V., Bercaw, R. J., Loomis, G. W., Hoyt, K. P., Avizinis, E. J., Amador, J. A. (2016). Accuracy of rapid tests used for analysis of advanced onsite wastewater treatment system effluent. Water, Air, \& Soil Pollution, 227, 310.

Murphy, L., Cahn, M. \& Smith, R. (2014). Accuracy of test strips for assessing nitrate concentration in soil and water. Salinas Valley Agriculture. Available at: http://ucanr.edu/blogs/blogcore/postdetail.cfm?postnum=13140

Oakley, S. M., Gold, A. J., Oczkowski, A. J. (2010). Nitrogen control through decentralized wastewater treatment: Process performance and alternative management strategies. Ecological Engineering, 36, 1520-1531.

Sims, J. R., \& Jackson, G. D. (1971). Rapid analysis of soil nitrate with chromotropic acid. Soil Science Society of America Journal, 603-606.

Sohail, M., \& Adeloju, S. B. (2016). Nitrate biosensors and biological methods for nitrate determination. Talanta, 153, 83-98.

Taylor, A., \& Kerr, G. (1941). The distribution of energy in the visible spectrum of daylight. Journal of the Optical Society of America, 31, 3-8.

Valiela, I., Foreman, K., LaMontagne, M., Hersh, D., Costa, J., Peckol, P., \& Brawley, J. (1992). Couplings of watersheds and coastal waters: Sources and consequences of nutrient enrichment in Waquoit Bay, Massachusetts. Estuaries, 15(4), 443-457.

Weatherburn, M. (1967). Phenol-hypochlorite reaction for determination of ammonia. Analytical Chemistry, 39(8), 971-974. 